\title{
Variáveis de Contexto e Respostas à Mudança Organizacional: Testando o Papel Mediador das Atitudes.
}

\author{
Vanessa de Fátima Nery ${ }^{1}$ \\ Elaine Rabelo Neiva \\ Universidade de Brasília
}

\begin{abstract}
RESUMO - No contexto atual, a regra nas organizações passou a ser a mudança em ritmo cada vez mais acelerado, demandando constante assimilação por parte dos empregados. Por isso, este estudo investiga a mediação das atitudes na relação entre contexto e respostas à mudança organizacional. Para testar esse modelo de mediação foi realizado um estudo transversal quantitativo em duas organizações públicas. Os 981 participantes responderam três escalas. Foram realizadas análises de confiabilidade das medidas e análises de regressão para o teste do modelo de mediação. O modelo foi parcialmente corroborado, indicando a influência da atitude e do contexto nas respostas comportamentais à mudança organizacional. Este estudo tem implicações teóricas e metodológicas para o campo do comportamento organizacional. A contribuição prática se refere a um aumento da compreensão a respeito dos fatores que facilitam o sucesso das mudanças organizacionais.
\end{abstract}

Palavras-chave: mudança organizacional, contexto de mudança organizacional, atitudes frente à mudança organizacional, resistência, comportamentos de apoio à mudança

\section{Context Variables and Responses to Organizational Change: Testing the Mediating Role of Attitudes}

\begin{abstract}
In the current context, increasingly fast-paced changes have become the rule in organizations, demanding constant assimilation on the part of employees. Therefore, this study investigates the mediation of attitudes in the relationship between context and responses to organizational change. To test the mediation model a quantitative cross-sectional study was conducted in two public organizations. The 981 participants completed three scales. Analyses of reliability of the scales were realized while regression analysis was used to test the mediation model. The model was partially confirmed, indicating the influence of the context on attitude and behavioral responses to organizational change. This study has theoretical and methodological implications for the field of organizational behavior. The practical contribution refers to an increased understanding about the factors that facilitate the success of organizational change.
\end{abstract}

Keywords: organizational change, organizational context change, attitudes toward organizational change, resistance, behavior to support change

O contexto de mudança organizacional é um aspecto constantemente negligenciado nas pesquisas nacionais e internacionais sobre mudança (Pettigrew, Woodman, \& Cameron, 2001). Por isso, devido à sua influência nos indivíduos e no processo de mudança, o contexto de mudança é uma característica importante a ser investigada (Kalimo, Taris, \& Schaufeli, 2003). Dessa forma, este estudo pretende investigar as seguintes características do contexto de mudança: a frequência, o planejamento e preparação da mudança, o grau de incerteza psicológica (Rafferty \& Griffin, 2006), histórico, intensidade e as experiências anteriores com processos de mudança organizacional (Cunningham, 2006; Devos, Buelens, \& Bouckencooghe, 2007; Kalimo, Taris, $\&$ Schaufeli, 2003).

A literatura apresenta muitas especulações com poucos estudos empíricos que buscam identificar e compreender os aspectos da mudança que influenciam as atitudes e

1 Endereço para correspondência: Instituto de Psicologia, Universidade de Brasília, Campus Darcy Ribeiro, ICC Ala Sul, Sala AT-013, Asa Norte, Brasília, DF, Brasil. CEP: 70.910-900. E-mail: vanessa.nery@gmail.com os comportamentos dos indivíduos (Armenakis, Harris, Mossholder, \& Isabella, 2007; Oreg, Vakola, \& Armenakis, 2011; Rafferty \& Griffin, 2006; Vakola, Tsaousis, \& Nikolaou, 2004). Além disso, a literatura ressalta a necessidade de mais estudos que investiguem os efeitos que as atitudes frente à mudança organizacional causam nos empregados (Choi, 2011). De forma pioneira, em 2012 foi realizado um estudo abrangendo quatro organizações brasileiras que investigou a influência do contexto de mudança nas atitudes de empregados frente à mudança organizacional. Nesse estudo, os autores encontram relação significante entre os fatores de contexto e atitudes frente à mudança (Neiva, Goulart, Fussi, $\&$ Nery, 2012).

A literatura também aponta que os critérios utilizados para avaliar as mudanças são objetivos, relacionados à produtividade e ao resultado organizacional (Neiva \& Paz, 2012). Ao considerar que a implementação de mudanças organizacionais impõe aos empregados a adoção de novos comportamentos necessários, percebe-se que esses indicadores brutos não são suficientes para abranger o aspecto humano. Por isso, em um processo de implantação da mudança é necessário analisar que as iniciativas podem 
evocar respostas comportamentais tais como resistência e outros comportamentos de apoio (Armenakis \& Bedeian, 1999). Esses fatores também são indicadores do sucesso das mudanças implementadas.

Com o objetivo de investigar a relação existente entre o contexto de mudança, as atitudes e as respostas comportamentais à mudança, este estudo testou um modelo mediacional das atitudes na relação entre o contexto de mudança organizacional e as respostas comportamentais - de apoio e resistência.

\section{Contexto de Mudança Organizacional}

A mudança organizacional pode ser um processo de transformação, ou um evento que deve ser considerado em relação ao contexto institucional maior (Hatch \& Ehrlich, 1993), sendo importante especificar sua caracterização para investigá-la (Lima \& Bressan, 2003).

A literatura aponta as seguintes características que constituem o contexto de mudança: a frequência, a intensidade, o escopo, o planejamento da mudança e o grau de incerteza psicológica, a velocidade, o risco das mudanças, as histórias anteriores com processos de mudança e perspectivas futuras com novos processos de mudança. Essas características contextuais influenciam as crenças, atitudes e intenções dos membros organizacionais (Cunningham, 2006; Devos, Buelens, \& Bouckencooghe, 2007; Rafferty \& Griffin, 2006; Self, Armenakis, \& Schraeder, 2007).

O contexto de mudança influencia a percepção dos indivíduos. Assim, quando o contexto é caracterizado por mudanças muito intensas ou frequentes pode haver consequências indesejadas, como, por exemplo: ansiedade, emoções negativas, incerteza e ambiguidade entre os empregados (Devos et al., 2007; Vakola et al., 2004).

O planejamento de mudança é definido como a percepção dos indivíduos a respeito da preparação feita pela organização ocorrida antes da implementação da mudança. A literatura indica que quando a organização planeja as ações relacionadas à mudança com antecedência, os empregados tendem a percebê-la como menos ameaçadora (Cunningham, 2006; Devos et al., 2007).

Em 2012, Neiva e Nery validaram a única escala nacional que caracteriza o contexto de mudança organizacional, por isso, foi adotada neste estudo para acessar a variável antecedente. É importante ressaltar que não foram encontrados na literatura outros instrumentos que se dediquem a tal propósito (Lima \& Bressan, 2003; Neiva \& Paz, 2012).

\section{Atitudes frente à mudança organizacional}

Atitudes frente às mudanças organizacionais consistem em cognições pessoais, reações afetivas e tendências comportamentais relacionadas aos aspectos da mudança empresarial. Assim, as atitudes frente à mudança organizacional são formadas a partir de uma avaliação, positiva ou negativa, que um empregado desenvolve a respeito das mudanças implementadas pela organização (Lines, 2005).

Estudiosos demonstram muito interesse sobre as atitudes frente à mudança (Bouckenooghe, 2010). Diversos estudos abordam o processo de formação de atitudes (Lau \& Woodman, 1995) e a forma de investigá-las (Lines, 2005; Vakola et al., 2004). As atitudes dos indivíduos frente à mudança são relevantes para o sucesso das iniciativas de mudança, pois desde os primeiros contatos com as ações de mudança os indivíduos de uma organização questionarão a forma como a mudança irá impactar seus trabalhos, e a possibilidade de sucesso organizacional (Armenakis, et al., 2007).

As atitudes dos empregados frente à mudança organizacional são aspectos críticos para o sucesso dos processos de mudança organizacional porque predispõem os indivíduos a agir de determinada forma em relação a um objeto (Armenakis et al., 2007; Vakola et. al., 2004). Valley e Thompson (1998) afirmam que atitudes moldam sentimentos das pessoas e suas percepções em relação à mudança, predispondo a ocorrência de comportamentos relacionados à atitude. Isso significa que a ocorrência de atitudes positivas - atitudes de aceitação - durante o processo de mudança facilitaria a ocorrência de comportamentos adaptativos do indivíduo às mudanças e aumentaria a probabilidade de sucesso das iniciativas. Em contrapartida, as atitudes negativas à mudança - como, por exemplo, o temor e o ceticismo - podem ter o efeito oposto, diminuindo a probabilidade de comportamentos de apoio dos empregados e do sucesso das intervenções (Neiva \& Paz, 2012).

Em 2005, Neiva, Ros e Paz validaram uma escala para mensurar as atitudes frente à mudança organizacional, composta por três fatores (ceticismo, temores e aceitação) que representam as atitudes típicas apresentadas pelos indivíduos em situação de mudança organizacional. As atitudes frente à mudança organizacional são investigadas nesse estudo como variável mediadora, e serão acessadas pela escala citada.

\section{Respostas Emocionais e Comportamentais à Mudança Organizacional}

As reações à mudança organizacional têm atraído o interesse de práticos e acadêmicos devido à sua influencia nos empregados (Devos et al., 2007) e no sucesso do processo de mudança (Oreg et al., 2011). Contudo, existem poucas pesquisas sobre os fatores que influenciam as reações dos empregados e sobre seu impacto nos comportamentos dos empregados, nas relações sociais de trabalho e nos resultados organizacionais.

As primeiras experiências e informações a respeito da mudança são suficientes para provocar emoções como a frustração, o entusiasmo ou o medo nos empregados (Lines, 2005). Quando a mudança organizacional aumenta a carga de trabalho ou retira vantagens dos empregados, as reações negativas ocorrem quase que imediatamente. Ao interpretarem a mudança como ameaça, os empregados geralmente experimentam sofrimento, ansiedade e estresse. A mudança organizacional tende a ser interpretada de forma positiva quando elimina rotinas aborrecedoras e potencializa 
as oportunidades de crescimento e desenvolvimento dos empregados, gerando excitação e motivação (Kruglanski, Higgins, \& Capozza, 2007).

As mudanças radicais que envolvem downsizing e consequente perda de empregos tendem a tornar os empregados mais resistentes. Ainda que a mudança radical seja reconhecida pelos empregados como necessária à organização, pode ser percebida como ameaça gerando sentimentos de incerteza a respeito da estabilidade no cargo. E, por terem consequências rápidas, são frequentemente interpretadas como traumáticas, gerando pouco comprometimento, provocando resistência, ressentimentos, mágoas e até mesmo boicotes (Kruglanski et al., 2007). Contudo, as respostas à mudança devem ser compreendidas como uma ocorrência normal, quer sejam positivas ou negativas (Battilana \& Casciaro, 2013; Bovey \& Hede, 2001).

As emoções negativas tornam os indivíduos mais propensos a pensamentos de curto prazo e a resultados organizacionais indesejados. Já as emoções positivas podem influenciar a adaptação dos empregados às novas situações de trabalho. Assim, os empregados com emoções ou afetos positivos tendem a ter atitudes menos cínicas em relação à mudança. Nesse sentido, reações e emoções positivas no contexto de mudança facilitariam o sucesso dos programas de mudança e a redução da resistência aos programas (Baumeister, Gailliot, DeWall, \& Oaten, 2006).

Considerando que as reações à mudança podem variar desde a imediata adesão até a resistência total (Lima \& Bressan, 2003) e que podem ser classificadas nas dimensões comportamental, cognitiva e afetiva (Piderit, 2000), o enfoque às reações à mudança nesse trabalho foi classificado na dimensão comportamental considerando as seguintes respostas comportamentais: os comportamentos de apoio às mudanças e a resistência.

\section{Comportamentos de apoio à mudança organizacional}

A manifestação de comportamentos de apoio à mudança organizacional é a reação positiva mais desejada pelas empresas, devido à criticidade da cooperação humana para o sucesso das mudanças organizacionais (Oreg, 2003). Para este estudo, consideram-se como comportamentos de apoio à mudança a emissão de comportamentos que promovam e facilitem a implantação da mudança (Avey, Wernising, \& Luthans, 2008; Devos et al., 2007).

Vários são os construtos que abordam respostas dos empregados em relação ao processo de mudanças, contudo poucos abordam diretamente as respostas comportamentais (Kim, Hornung, \& Rosseau, 2011). Meyer e colaboradores (Hercovith \& Meyer, 2002; Meyer, Srinivas, Lal, \& Topolnytsky, 2007) estudaram os comportamentos de apoio à mudança como consequência do comprometimento com a mudança e apresentaram três dimensões para as respostas comportamentais positivas dos indivíduos durante o processo de mudanças: condescendência (apoio mínimo e relutante); cooperação e entusiasmo (contribuições excepcionais e promoção da mudança para os outros).

Kim et al. (2011) defendem que os comportamentos de apoio à mudança se referem ao papel positivo e ativo que os empregados podem ter no apoio à mudança organizacional e os definem como ações dos empregados que se engajam no processo de mudanças, participando ativamente para facilitar e contribuir com a mudança planejada. Os autores citados argumentam que enfatizar os comportamentos atuais, as contribuições ativas e as ações coletivas produz maiores resultados de pesquisa que a ênfase em estados psicológicos, respostas passivas e individuais.

Assim, o presente estudo adota tal perspectiva enfatizando os comportamentos de oposição e de participação ativa nas mudanças como forma de distinguir papéis diferenciados no processo e abordar ações mais facilmente caracterizadas.

Alguns estudos identificaram fatores que facilitam os comportamentos de apoio à mudança organizacional, como por exemplo, a participação dos trabalhadores durante o processo de implementação de mudanças; a avaliação que os empregados fazem do programa; e a percepção que desenvolvem a respeito da utilidade da intervenção (Coyle-Shapiro, 1999; Zammuto \& O' Connor, 1992). Os empregados estão mais propensos a apoiar as mudanças organizacionais quando existe um sentimento de confiança e de apego à organização ou quando a organização demonstra uma preocupação com a qualidade de vida no trabalho (Cunningham, 2006; Huy, 2002). Dessa forma, os indivíduos se perceberiam valorizados e respeitados no processo de mudanças.

A presença de um líder que transmita confiança é um fator crítico que facilita a adesão dos empregados ao processo de mudança. Além disso, a literatura ressalta a importância da comunicação durante o processo de mudança, indicando que os empregados devem ter papel ativo, entendendo porque ela está acontecendo e quais os benefícios que eles terão. A presença de pessoas jovens na organização é outro aspecto que facilita o processo de mudança, por serem mais conscientes das exigências do mercado, agregarem novas ideias para a empresa e serem mais receptivos às mudanças, oferecem menos resistência do que os empregados que têm mais idade (Lima \& Bressan, 2003).

O impacto dos aspectos psicológicos dos empregados nas atitudes e comportamentos foi investigado por Avey et al. (2008). Os autores identificaram que (1) aspectos psicológicos - tais como esperança, otimismo, eficácia e resiliência - estão relacionados positivamente às emoções positivas, que por sua vez estão relacionadas às atitudes e aos comportamentos relevantes para a mudança organizacional; (2) o nível de conscienciosidade interage com os aspectos psicológicos na predição das emoções positivas; e (3) as emoções positivas medeiam o relacionamento entre os aspectos psicológicos, as atitudes e os comportamentos.

\section{Resistência à mudança organizacional}

A literatura não apresenta consenso a respeito da definição sobre resistência à mudança. Piderit (2000) ressalta a ambiguidade do construto resistência à mudança e sugere a composição tripartite: a cognitiva, a dimensão afetiva e a dimensão comportamental. A abordagem cognitiva explica a resistência de três formas: a primeira a considera como um processo natural e normal gerada por crenças distorcidas ou 
pela tendência dos indivíduos a avaliarem situações usando categorias extremas; a segunda aborda a resistência como a intenção de resistir, como o conjunto composto da percepção do impacto da mudança, de ideias irracionais e afetos (Bovey \& Hede, 2001); a terceira considera a resistência como uma reação emocional negativa ativada pelas inconsistências e discrepâncias entre os esquemas cognitivos dos indivíduos e os atuais propostos pelo processo de mudança (George \& Jones, 2001).

Em uma revisão de literatura, Bouckenooghe (2010) identificou que a maioria das 14 definições de resistência considera o componente intencional/comportamental como uma força contrária que impede o sucesso da implementação da mudança e promove manutenção do status quo. Para este estudo, considerou-se a definição de resistência à mudança como a emissão de comportamentos de oposição ao processo de mudança, declarados ou não (Avey et al., 2008; CoyleShapiro, 1999, Devos et al., 2007).

A resistência à mudança é tratada pela literatura como um fator crítico para o sucesso da mudança (Trader-Leigh, 2002; Vakola et al., 2004). A ocorrência de comportamentos de resistência provoca efeitos negativos para a organização: atraso no início do processo de mudança, demora e ineficiência imprevistas na etapa de implantação, custos adicionais, além de ações de sabotagem ao processo de mudança (Eilam \& Shamir, 2005).

Apesar da criticidade, ainda há muito a se descobrir para a compreensão apropriada desse fenômeno (Bouckenooghe, 2010; Fischer \& Lima, 2005). Parte das publicações sobre o tema indica a resistência como uma das principais barreiras ao sucesso das transformações organizacionais (Piderit, 2000) e prescreve ações para diminuir o impacto das resistências à mudança organizacional. Contudo, a resistência à mudança não deve ser compreendida como um obstáculo a ser superado, mas como elemento inerente às transformações cognitivas que ocorrem durante a mudança (Isabella, 1990).

Valley e Thompson (1998), em um estudo longitudinal, identificaram que a resistência dos empregados possui bases relacionais e individuais e exerce influência nas relações de poder durante o processo de mudança e que a resistência é maior quando os empregados não são envolvidos no processo de mudança. Ashfort e Mael (1998) também apresentaram uma proposta de classificação dos comportamentos de resistência em autorizada e não autorizada, além de orientada e difusa. Tal classificação envolve desde comportamentos de manifestação verbal de desacordo, críticas aos gerentes e agentes de mudança, até sabotagem às intervenções realizadas.

A resistência consciente e racional pode ser muito mais útil do que a aceitação incondicional e inquestionável (Ford, Ford, \& D'Amelio, 2008). A literatura indica casos em que a resistência auxilia a aperfeiçoar as ações planejadas, evidenciando os aspectos que foram planejados inadequadamente ou negligenciados pelos agentes de mudanças. Dessa forma é possível utilizar a resistência para alterar componentes da mudança, tornando-a mais compatível à organização (Lima Filho, Sproesser, \& Hoffman, 2005).

Estudos indicam que a resistência varia de intensidade conforme o envolvimento, participação e conhecimento dos empregados sobre o processo de mudança (Armenakis \&
Bedeian, 1999). A intensidade da resistência à mudança pode variar em função de características pessoais dos indivíduos, do impacto negativo das mudanças, das exigências e novidades que envolvem, do sofrimento que causa a membros da organização (Lima \& Bressan, 2003).

As representações cognitivas (crenças, atitudes) individuais são consideradas como fatores que interferem no sucesso da mudança (Hodgkinson \& Healey, 2008). A literatura enfatiza a importância das atitudes nas respostas dos indivíduos frente à mudança (Valley \& Thompson, 1998), além de indicar que a resistência é iniciada a partir das atitudes negativas. Para investigar essa relação, as atitudes frente às mudanças é a variável mediadora desse estudo.

Conforme afirmado anteriormente, os processos de mudança organizacionais são frequentes. Considerando-se os comportamentos decorrentes dos diversos processos de mudança organizacional, formulou-se o seguinte problema de pesquisa: " $O$ contexto interno de mudança e as atitudes frente à mudança influenciam as reações comportamentais dos colaboradores?". O objetivo desse estudo foi testar a relação de mediação das atitudes frente à mudança entre variáveis de contexto, de conteúdo e de processo e os comportamentos de apoio à mudança organizacional e resistência.

A literatura indica que quando a organização planeja as ações relacionadas à mudança com antecedência, os empregados tendem a percebê-la como menos ameaçadora (Cunningham, 2006; Devos et al., 2007). Por isso, foi elaborada a hipótese (H1): o planejamento e o preparo da mudança predizem positivamente os comportamentos de apoio à mudança organizacional e negativamente os comportamentos de resistência. A literatura indica que quanto mais ameaçadora é a mudança, mais os empregados terão comportamentos negativos a respeito da mudança (Bordia, Restubog, Jimmieson, \& Irmer, 2011), o que subsidia a segunda hipótese $(\mathrm{H} 2)$, que presume que o grau de risco prediz os comportamentos de apoio à mudança organizacional e resistência. Valley e Thompson (1998) e Choi (2011) afirmam que atitudes moldam sentimentos das pessoas e suas percepções em relação à mudança, predispondo a ocorrência de comportamentos relacionados à atitude. Isso significa que a ocorrência de atitudes positivas - atitudes de aceitação ao processo de mudança, facilitaria a ocorrência de comportamentos de apoio às mudanças, aumentando a probabilidade de sucesso das iniciativas. Em contrapartida, atitudes negativas à mudança - como, por exemplo, o temor e o ceticismo - poderiam ter o efeito oposto, diminuindo a probabilidade da ocorrência de comportamentos de apoio e aumentando a resistência à mudança dos empregados, diminuindo o sucesso da mudança (Neiva \& Brito, 2008; Neiva \& Paz, 2012). Com base nessa literatura, foram definidas as hipóteses: (H3) as atitudes de temor e ceticismo predizem o comportamento de resistência, (H4) a atitude de aceitação prediz o comportamento de apoio à mudança organizacional.

Finalmente, foi elaborada a última hipótese deste estudo, (H5) as atitudes frente à mudança medeiam a relação entre planejamento e preparo e grau de risco e os comportamentos de apoio à mudança organizacional e resistência. Assim seria possível testar o modelo de mediação que ainda não havia sido investigado em estudos anteriores. 
As variáveis antecedentes do estudo são planejamento e preparo para a mudança e grau de risco. As variáveis mediadoras são as atitudes frente à mudança, e as variáveis critério são os comportamentos de apoio às mudanças $\mathrm{e}$ resistência.

\section{Método}

\section{Descrição das Organizações}

As análises foram realizadas com empregados e prestadores de serviço de duas organizações públicas do Setor Elétrico Brasileiro. A organização 1 é uma sociedade anônima de economia mista que atua na área de geração e transmissão de energia elétrica com sede no Distrito Federal e nove unidades regionais. A organização 2 é uma sociedade de economia mista que atua na distribuição e comercialização de energia elétrica.

\section{Descrição das Mudanças Organizacionais}

As organizações estudadas vivenciaram eventos ligados à mudança organizacional, e para facilitar a descrição das mudanças consideradas relevantes, optou-se por registrálas com base no conceito de contexto organizacional apresentado no estudo. Como visto em Armenakis e Bedeian (1999), o contexto externo inclui fatores como regulação governamental, avanços tecnológicos e forças que modelam a competição de mercado; e o contexto interno, por sua vez, inclui fatores como o grau de especialização do trabalho em relação a determinada tecnologia; a lentidão organizacional; e as experiências com mudanças anteriores. Foi constatado, após levantamento bibliográfico e documental, que as organizações passaram por reposicionamento estratégico a partir de pressões governamentais, incluindo mudança de missão e marca, implantação de um novo plano de cargos e salários com regras mais rígidas de progressão funcional, implantação de uma universidade corporativa, implantação do programa de avaliação de desempenho por resultados e competências, implantação de programa de desenvolvimento de potenciais líderes.

\section{Amostra}

A organização 1 possui 3800 empregados e 164 prestadores de serviço. Foi feita uma amostragem randômica estratificada, na qual os empregados e prestadores foram separados em estrados baseados nos 11 estados da federação. Foram convidados a participar da pesquisa 1600 empregados e 92 prestadores de serviço. Mas apenas 775 empregados e 51 prestadores responderam às escalas. A organização 2 possui 298 empregados, foram selecionados 130 participantes da pesquisa, mas efetivamente 55 responderam às escalas. A maioria dos participantes possui formação de, no mínimo, nível superior completo $(37,3 \%)$ e pertencem ao sexo masculino (74,5\%). Quanto à função exercida, $46 \%$ atuam na área fim das organizações, e 39,6\% na área meio.

A amostra foi distribuída em 11 estados da federação (AC, AM, AP, DF, MA, MT, PA, RR, RO, SP, TO). O Acre obteve o maior percentual de respondentes $(66,15 \%)$, seguido pelo Mato Grosso $(64,60 \%)$ e Distrito Federal $(62,25 \%)$. Os estados com menos respondentes foram São Paulo $(25 \%)$ e Amazonas (33,33\%).

\section{Instrumentos}

Neste estudo foram aplicados três instrumentos. Os três utilizam a escala do tipo Likert de 11 pontos que variava de 0 (discordo totalmente) a 10 (concordo totalmente).

O primeiro instrumento aplicado, a Escala de Contexto de Mudança Organizacional (Neiva \& Nery, 2012), possui estrutura bifatorial. $\mathrm{O}$ fator planejamento, preparação e experiências anteriores à mudança - PPEAM diz respeito às percepções dos empregados sobre as preparações que ocorreram antes da implementação das mudanças, como, por exemplo, o histórico e a frequência de mudanças e as experiências anteriores de sucesso ou fracasso. Já o fator risco e impacto das mudanças relaciona-se à percepção dos empregados sobre os aspectos decorrentes da implantação da mudança que podem afetar tanto a organização quanto o próprio indivíduo. A Escala de Contexto de Mudança Organizacional possui 21 itens com cargas fatoriais acima de 0,32 e alfas de Cronbach acima de 0,74. Exemplo de item: "A organização passou por muitas mudanças nos últimos anos."

O segundo instrumento é a escala de atitudes frente à mudança organizacional, validada por Neiva et al. (2005), que acessa crenças, comportamentos e sentimentos dos indivíduos durante os processos de mudança organizacional, em três fatores: ceticismo, temor e aceitação. O ceticismo abrange crenças e sentimentos de descrédito relacionados ao processo de mudança. O temor é caracterizado pelo medo de perder poder, benefícios ou das incertezas vivenciadas pelos indivíduos. Atitudes de aceitação se relacionam às crenças, sentimentos e intenções comportamentais que fazem uma avaliação positiva da mudança organizacional. Este instrumento é composto por 46 itens, cargas fatoriais acima de 0,30 e Alfa de Cronbach acima de 0,85. Exemplo de item: "As pessoas costumam negar que a mudança vai acontecer."

O terceiro instrumento, que acessou as reações comportamentais à mudança (Nery \& Neiva, 2012), possui estrutura bifatorial e seus 19 itens avaliam a ocorrência de respostas comportamentais de apoio e resistência à mudança. $\mathrm{O}$ fator comportamentos de apoio à mudança abrange a emissão de comportamentos que são desejados pelo processo de mudanças, tais como flexibilidade, abertura ao processo, aceitação, defesa da mudança. $\mathrm{O}$ fator resistência avalia comportamentos de oposição ao processo de mudança, declarados ou encobertos. As cargas fatoriais possuem valores acima de 0,40 e Alfa de Cronbach acima de 0,90. Exemplo de item: "Os empregados se comportam de maneira favorável à mudança." 


\section{Coleta de dados}

A coleta de dados estruturada foi realizada no software online Lime Survey. Os sujeitos selecionados foram convidados a participar da pesquisa por meio de um e-mail que continha um texto explicativo, o endereço online da pesquisa e a senha para acesso ao questionário. Ao acessar a pesquisa, os sujeitos eram apresentados a um termo de consentimento esclarecendo a respeito da confidencialidade e tratamento dos dados.

\section{Análise de dados}

Após a realização da pesquisa, o banco de dados recebeu os tratamentos necessários de preparação para as análises estatísticas. Em seguida foi realizada análise dos eixos principais (PAF) para confirmação da estrutura fatorial.

Foram verificados os pressupostos das análises estatísticas multivariadas. As investigações dos efeitos de mediação foram realizadas utilizando regressões hierárquicas, para confirmar o modelo procedeu-se à execução do teste Sobel, conforme proposto por Baron e Kenny (1986). Também foi realizada uma equação estrutural para teste geral do ajuste do modelo.

Este estudo utilizou apenas uma fonte de dados. Assim, questões sobre a influência da variância do método comum sobre os resultados do estudo foram levantadas. Por isso, foi realizada a análise fatorial confirmatória por Equações estruturais para comparar diferentes modelos (Bentler, 1989). Os resultados indicaram que o modelo de um único fator não apresentou ajuste aceitável $(N F I=0,46 ; C F I=0,39$ e $N N F I=0,49)$. O ajuste dos modelos fatoriais confirmatórios das outras variáveis perceptuais foi considerado bastante satisfatório $(N F I=0,96 ; C F I=0,95$ e $N N F I=0,94)$. Portanto, foi concluído que a variância do método comum sozinha não explica os resultados.

\section{Resultados}

Foram realizadas análises descritivas dos fatores, cujos dados são apresentados na Tabela 1. Esses índices demonstram o comportamento das variáveis do estudo, indicando que as respostas dos participantes concentraram- se, em geral, acima do ponto médio da escala, com alto valor de desvio padrão.

Para o teste do modelo, foram realizadas diversas análises de regressão, de acordo com os critérios de mediação propostos por Baron e Kenny (1986). As análises foram realizadas de forma separada, mas os resultados serão apresentados de forma consolidada, na Tabela 2 .

Ao comparar os coeficientes b e Beta, na relação entre a variável antecedente PPEAM e as variáveis critério comportamentos de apoio às mudanças e resistência, é possível verificar que são estatisticamente diferentes de zero, sendo que para a variável comportamentos de apoio às mudanças o modelo possui um bom poder explicativo $\left(R^{2}=0,40\right)$. Ao analisar os coeficientes b e Beta da variável risco em relação às variáveis comportamentos de apoio às mudanças e resistência, também são obtidos valores estatisticamente diferentes de zero (Tabela 2). De acordo com esses resultados, é possível afirmar que a primeira condição para análise da mediação foi satisfeita.

O sumário das análises para verificar o segundo critério proposto por Baron e Kenny (1986) são apresentados de forma consolidada, na Tabela 3. Os valores dos coeficientes b e Beta nas relações entre as variáveis antecedentes e as mediadoras são significativamente diferentes de zero. De acordo com os dados, também é possível afirmar que o modelo com melhor poder explicativo é da variável PPEAM, predizendo $57 \%$ da aceitação $\left(R^{2}=0,57 ; p<0,01\right)$. De acordo com esses resultados, é possível afirmar que o segundo critério foi atingido.

Os dados apresentados na Tabela 2 ilustram que as variáveis critério comportamentos de apoio às mudanças e resistência são explicadas pelas três variáveis de atitudes frente à mudança de forma significativa. Os dados apresentados na Tabela 2 demonstram que a variável PPEAM obteve decréscimo nos valores dos coeficientes $B$ e Beta reduzidos pela presença das variáveis mediadoras aceitação, temor e ceticismo que foram incluídas nas análises com as variáveis critérios. De acordo com os dados, os modelos com maior valor de predição são entre as variáveis PPEAM, aceitação e comportamentos de apoio às mudanças $\left(R^{2}=0,52\right.$; $p<0,01)$, e as variáveis PPEAM, ceticismo e resistência $\left(R^{2}=0,54 ; p<0,01\right)$. Na relação entre a variável risco e as variáveis critério, percebe-se que os valores dos coeficientes $B$ e Beta reduzidos pela presença das variáveis mediadoras aceitação, temor e ceticismo quando foram incluídas nas

Tabela 1. Correlações, Média e Desvio padrão das variáveis do estudo

\begin{tabular}{|c|c|c|c|c|c|c|c|c|}
\hline Variáveis & 1 & 2 & 3 & 4 & 5 & 6 & Média & $\begin{array}{l}\text { Desvio } \\
\text { Padrão }\end{array}$ \\
\hline 1. (Co) PPEAM & & & & & & & 6,04 & 1,66 \\
\hline 2. (Co) Risco & $-0,168^{*}$ & & & & & & 5,96 & 1,74 \\
\hline 3. Aceitação & $0,767 *$ & $-0,171 *$ & & & & & 6,96 & 1,53 \\
\hline 4. Temor & $-0,254^{*}$ & $0,437 *$ & $-0,203^{*}$ & & & & 7,05 & 1,62 \\
\hline 5. Ceticismo & $-0,501 *$ & $0,365^{*}$ & $-0,520 *$ & $0,492 *$ & & & 4,33 & 1,82 \\
\hline 6. Comportamento de apoio à mudança & $0,640 *$ & $-0,202 *$ & $0,714^{*}$ & $-0,319^{*}$ & $-0,547 *$ & & 5,8 & 1,61 \\
\hline 7. Resistência & $-0,314^{*}$ & $0,308^{*}$ & $-0,358 *$ & $0,405^{*}$ & $0,719 *$ & $-0,550 *$ & 3,86 & 1,82 \\
\hline
\end{tabular}

Nota. ${ }^{*} \mathrm{p}<0,01$; 
Tabela 2. Sumário das análises de regressão

\begin{tabular}{|c|c|c|c|c|c|c|c|}
\hline \multirow{3}{*}{ Variável antecedente } & \multicolumn{6}{|c|}{ Variável critério } & \multirow{3}{*}{$\begin{array}{l}\text { (teste de } \\
\text { sobel) Z }\end{array}$} \\
\hline & \multicolumn{3}{|c|}{ Comportamento de apoio à mudança } & \multicolumn{3}{|c|}{ Resistência } & \\
\hline & $\mathrm{R}^{2}$ & $\mathrm{~B}$ & $\beta$ & $\mathrm{R}^{2}$ & $\mathrm{~B}$ & $\beta$ & \\
\hline PPEAM & $0,401 *$ & $0,451 *$ & $0,633^{*}$ & $0,094 *$ & $-0,276^{*}$ & $-0,307 *$ & \\
\hline Risco & $0,030^{*}$ & $-0,297 *$ & $-0,173 *$ & $0,096^{*}$ & $0,670^{*}$ & $0,310 *$ & \\
\hline Aceitação & $0,486^{*}$ & $0,517^{*}$ & $0,697^{*}$ & $0,078^{*}$ & $-0,262 *$ & $-0,279 *$ & \\
\hline Temor & $0,079 *$ & $-0,304 *$ & $-0,281^{*}$ & $0,145^{*}$ & $0,522 *$ & $0,380 *$ & \\
\hline Ceticismo & $0,260 *$ & $-0,256^{*}$ & $-0,510^{*}$ & $0,538 *$ & $0,466^{*}$ & $0,733 *$ & \\
\hline \multirow[t]{2}{*}{ PPEAM X aceitação } & $0,521 *$ & $0,204 *$ & $0,289 *$ & $0,099 *$ & $-0,202 *$ & $-0,226^{*}$ & $-8,475^{*}$ \\
\hline & & $0,354^{*}$ & $0,477 *$ & & $-0,100 * *$ & $-0,107 * *$ & \\
\hline \multirow[t]{2}{*}{ PPEAM X temor } & $0,442 *$ & $0,438 *$ & $0,620 *$ & $0,194 *$ & $-0,205^{*}$ & $-0,229 *$ & $-6,855^{*}$ \\
\hline & & $-0,143 *$ & $-0,133 *$ & & $0,446^{*}$ & $0,325^{*}$ & \\
\hline \multirow[t]{2}{*}{ PPEAM X ceticismo } & $0,479 *$ & $0,374^{*}$ & $0,530^{*}$ & $0,540^{*}$ & $0,043 *$ & $0,048^{*}$ & $-10,645^{*}$ \\
\hline & & $-0,131^{*}$ & $-0,261 *$ & & $0,481^{*}$ & $0,756^{*}$ & \\
\hline \multirow[t]{2}{*}{ Risco X aceitação } & $0,494 *$ & $-0,152 *$ & $-0,089 *$ & $0,154^{*}$ & $0,600^{*}$ & $0,278^{*}$ & 1,58 \\
\hline & & $0,509^{*}$ & $0,687^{*}$ & & $-0,231^{*}$ & $-0,245^{*}$ & \\
\hline \multirow[t]{2}{*}{ Risco X temor } & $0,083^{*}$ & $-0,111 \mathrm{t}$ & $-0,065 t$ & $0,171^{*}$ & $0,387^{*}$ & $0,179 *$ & $6,105^{*}$ \\
\hline & & $-0,275^{*}$ & $-0,254^{*}$ & & $0,418^{*}$ & $0,305^{*}$ & \\
\hline Risco $\mathrm{X}$ ceticismo & $0,261 *$ & $0,047 * *$ & $0,027 * *$ & $0,539 *$ & $0,068 * *$ & $0,031 * *$ & $3,61 *$ \\
\hline
\end{tabular}

Nota. $* * \mathrm{p}<0,01 ; * * \mathrm{p}<0,05 ; \mathrm{tp}<0,10$

análises. Na relação entre risco e comportamento de apoio à mudança, os valores dos coeficientes deixaram de ser significativos, sugerindo que a variável temor funciona como mediadora completa, pois reduziu o valor absoluto dos coeficientes de inclinação, tornando-os estatisticamente iguais a zero. Os dados indicam que o modelo de mediação contendo as variáveis risco, ceticismo e resistência tem alto valor explicativo $\left(R^{2}=0,53 ; p<0,01\right)$. Esses resultados indicam que a terceira condição foi atendida, considerando que as atitudes atuaram como mediadoras entre as variáveis antecedentes e critério. Para confirmar o modelo mediacional foi realizado o teste Sobel (Baron \& Kenny, 1986), cujos resultados são apresentados na Tabela 2 .

Os resultados do teste Sobel apresentados na Tabela 2 confirmam a mediação da atitude de aceitação na relação entre PPEAM e o comportamento de apoio à mudança, de forma significativa $(Z=25,72 ; p<0,001)$. A relação mediacional da atitude de aceitação na relação entre PPEAM e a resistência foi significativa $(Z=-8,47 ; p<$ $0,001)$. Os resultados apresentados na Tabela 2 indicam que as relações de mediação entre as variáveis risco, aceitação e comportamentos de apoio às mudanças e entre risco, aceitação e resistência não foram significativas, indicando que o teste Sobel não confirmou a mediação. As demais relações obtiveram resultados significativos, corroborando as relações mediacionais.

O modelo de relações apresentado pela pesquisa também apresentou bons índices de ajuste de acordo com o teste por equações estruturais. $\mathrm{O}$ modelo apresentado na Figura 1 possui ajuste em todos os índices: $\chi^{2}(98)=107,91, n s ; \chi^{2} / d f$ $=2,10 ; I F I=0,99 ; T L I=0,98 ; C F I=0,98 ; R M S E A=0,027$, $C I=0,011-.088, n s$.

\section{Discussão}

A primeira e a segunda hipóteses preconizaram que o planejamento e preparo das mudanças e o grau de risco predizem os comportamentos de apoio às mudanças e resistência. Os dados indicaram que a variável PPEAM explica mais a variável critério comportamentos de apoio às mudanças do que a variável resistência. Ou seja, indica que

Tabela 3. Sumário das análises de regressão entre as variáveis antecedentes e mediadoras

\begin{tabular}{|c|c|c|c|c|c|c|c|c|c|}
\hline \multirow{3}{*}{$\begin{array}{l}\text { Variáveis } \\
\text { antedentes }\end{array}$} & \multicolumn{9}{|c|}{ Variáveis mediadoras } \\
\hline & \multicolumn{3}{|c|}{ Aceitação } & \multicolumn{3}{|c|}{ Temor } & \multicolumn{3}{|c|}{ Ceticismo } \\
\hline & $R^{2}$ & $B$ & $\beta$ & $R^{2}$ & $\boldsymbol{B}$ & $\beta$ & $R^{2}$ & $B$ & $\beta$ \\
\hline PPEAM & $0,574 *$ & $0,722 *$ & $0,758^{*}$ & $0,062 *$ & $-0,163 *$ & $-0,248^{*}$ & $0,220 *$ & $-0,661 *$ & $-0,469^{*}$ \\
\hline Risco & $0,127^{*}$ & $-0,291 *$ & $-0,127 *$ & $0,179 *$ & $0,668^{*}$ & $0,423 *$ & $0,153 *$ & $1,325^{*}$ & $0,391 *$ \\
\hline
\end{tabular}

Nota. $* \mathrm{p}<0,01$ 


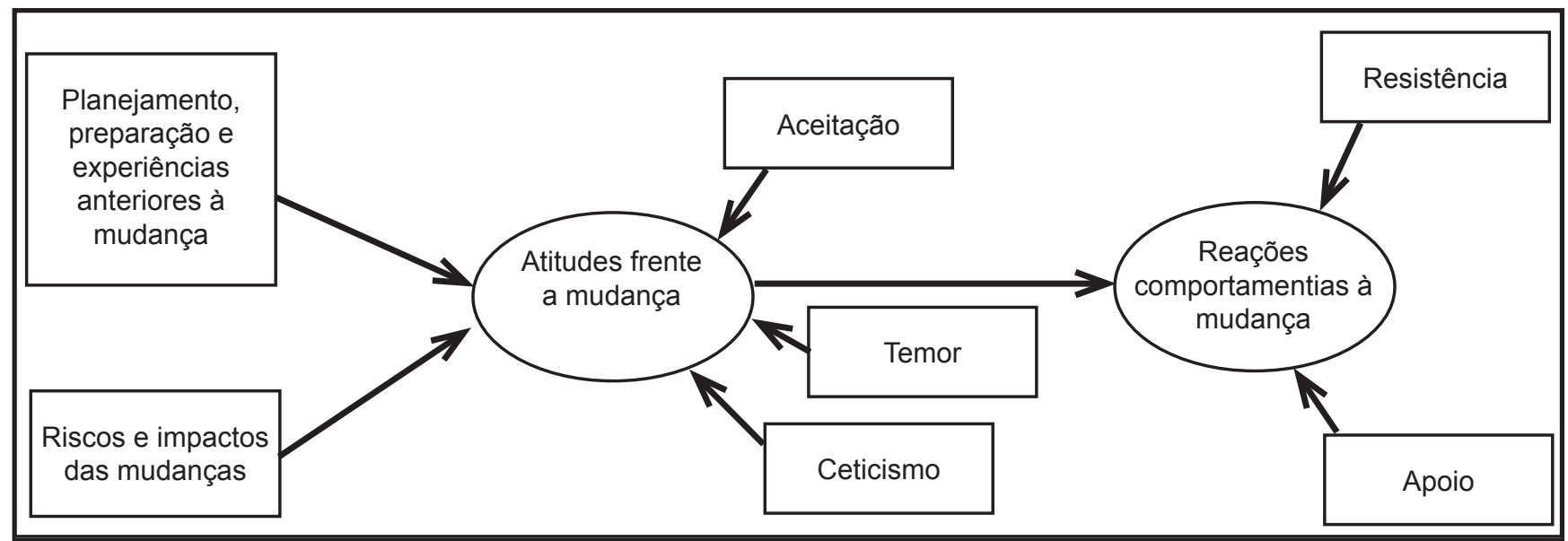

Figura 1 .Modelo testado por equações estruturais

a probabilidade de comportamentos de apoio às mudanças é significativamente maior quando os empregados percebem que a mudança é planejada e preparada pela organização. Esses dados corroboram a literatura que indica a importância do planejamento para o sucesso da mudança (Devos et al., 2007).

A variável risco e impacto das mudanças explica uma porção baixa, mas significativa, das variáveis comportamentos de apoio às mudanças e resistência. Em contrapartida, a percepção de risco da mudança não aumenta a probabilidade de engajamento. De acordo com esses resultados é possível afirmar que a hipótese 1 foi corroborada e a hipótese 2 foi fracamente corroborada. Estudos anteriores indicam que o grau de risco percebido no contexto de mudanças influencia tanto a abertura dos indivíduos para aceitar o processo de mudanças quanto a emissão dos comportamentos de apoio à mudança (Devos et al., 2007).

A terceira hipótese presumiu que as atitudes de temor e ceticismo predizem o comportamento de resistência. Os resultados apresentados na Tabela 2 corroboram a hipótese indicando que o ceticismo - a falta de crença, desconfiança - frente às ações de mudança explicam mais a resistência do que o temor. De acordo com os resultados, a segunda hipótese foi corroborada, e de acordo com a literatura, de fato há influência das atitudes negativas à mudança - temor e ceticismo - na ocorrência de comportamentos que dificultam a adaptação dos indivíduos às mudanças (Neiva \& Brito, 2008; Neiva \& Paz, 2012). Contudo, faz-se necessário realizar mais estudos que investiguem os efeitos que as atitudes frente à mudança organizacional causam nos empregados (Choi, 2011).

A quarta hipótese propôs que a atitude de aceitação prediz o comportamento de apoio à mudança. Os dados do presente estudo corroboram essa hipótese, indicando que a atitude de aceitação à mudança aumenta a probabilidade de comportamentos que facilitam a adaptação do indivíduo às mudanças, aumentando a probabilidade de sucesso das iniciativas, como foi apontado por Neiva e Paz (2012).

A quinta hipótese sugeriu que as atitudes frente à mudança medeiam a relação entre contexto de mudança e os comportamentos de apoio às mudanças e resistência. Contudo, os resultados do teste Sobel (Tabela 2) indicam que o modelo mediacional foi parcialmente corroborado. De acordo com os resultados, pode-se afirmar que a variável aceitação não é mediadora na relação entre risco e os comportamentos de respostas à mudança, sejam eles comportamentos de apoio às mudanças ou de resistência. Isso indica que o risco do contexto interno de mudanças não influenciou as atitudes nem as repostas à mudança nas organizações investigadas. É possível que esse resultado decorra da característica das organizações públicas deste estudo. Apesar dos empregados serem contratados pelo regime celetista, há uma percepção predominante de estabilidade, os empregados não percebem que a sua permanência na organização sofra ameaça. Assim, sugere-se que sejam investigadas organizações privadas para verificar se essa relação permanece em um contexto de mercado mais competitivo.

O papel de mediação parcialmente confirmado neste estudo é relevante para a literatura, pois corrobora a literatura indicando a influência das atitudes frente à mudança em facilitar o sucesso dos processos de mudança organizacional (Neiva \& Nery, 2012; Neiva \& Paz, 2012). Para a prática do Comportamento Organizacional, esse achado é bastante relevante, considerando que mudança organizacional é um fenômeno complexo e multifacetado, e grande parte da publicação se caracteriza pelos poucos dados empíricos e pela abundância das proposições prescritivas.

A mudança organizacional em larga escala só ocorre a partir da mudança no comportamento dos membros organizacionais (Santos, Neiva, \& Andrade-Melo, 2013). Por isso, as atitudes de aceitação à mudança que influenciam o comportamento de apoio à mudança podem ser melhor gerenciadas buscando reduzir o alto índice de fracasso das mudanças apontado pela literatura.

É importante destacar que as atitudes negativas frente à mudança são preditoras, mas não devem ser confundidas com resistência. As atitudes negativas deverão ser utilizadas apenas como indicativos de intenção comportamental, auxiliando ações de gerenciamento das comunicações a respeito da mudança.

A literatura indica que quando a resistência é bem gerenciada, o processo de mudança pode ser facilitado. Caso contrário, pode provocar estresse e mal-estar intolerável (Bortolotti, Souza Junior, \& Andrade, 2009). 


\section{Considerações Finais}

A literatura contém poucos estudos que investiguem os aspectos da mudança que influenciam as atitudes e os comportamentos dos indivíduos (Armenakis et al., 2007; Neiva et al., 2012; Oreg, Vakola, \& Armenakis, 2011; Rafferty \& Griffin, 2006; Vakola et al., 2004). O contexto da mudança organizacional é um tema frequentemente negligenciado nos estudos dessa área (Pettigrew \& Cameron, 2001). Pode-se afirmar que o presente estudo contribui para uma maior compreensão a respeito do papel do contexto de mudança nas atitudes e reações à mudança, pois investigou fatores intervenientes às reações à mudança, aspecto que ainda não tinha sido evidenciado pela literatura.

O campo do comportamento organizacional possui poucos estudos longitudinais. E, o tempo é uma questão importante para uma melhor compreensão do fenômeno da mudança. Por isso, faz-se necessário a realização de estudos que investiguem a mudança em delineamentos longitudinais (Lau \& Woodman, 1995). A triangulação de dados também é um aspecto crítico para abarcar a complexidade do fenômeno e conferir robustez para a área de estudos. Entretanto, não foi possível assimilar esses aspectos na realização deste estudo. Apesar das limitações metodológicas, considera-se que o presente trabalho atingiu seus objetivos principais e apontou importantes implicações práticas, teóricas e metodológicas. Recomenda-se que este estudo seja replicado em organizações de outros setores produtivos, buscando confirmar essa relação e possibilitar a generalização dos resultados.

Por fim, espera-se que o presente estudo contribua para a avaliação de mudanças organizacionais, bem como sirva de estímulo a outros pesquisadores interessados em investigar fatores que facilitem o sucesso das mudanças.

\section{Referências}

Avey, J. B., Wernsing, T. S., \& Luthans, F. (2008). Can Positive Employees Help Positive Organizational Change? Impact of Psychological Capital and Emotions on Relevant Attitudes and Behaviors. Journal of Applied Behavioral Science, 44, 48-70.

Armenakis, A. A., \& Bedeian, A. G. (1999). Organizational change: a review of theory and research in the 1990s. Journal of Management, 25, 293-315.

Ashford, B. E., \& Mael, F. (1998). The power of resistance: Sustaining valued identities. In R. M. Kramer \& M. A. Neale (Eds.), Power and influence in organizations (pp. 89-119). Thousand Oaks, CA: Sage.

Armenakis, A. A., Harris, S. G., Mossholder, K. W., \& Isabella, L. A. (2007). Readiness for Organizational Change: The Systematic Development of a Scale. The Journal of Applied Behavioral Science, 43, 232-255.

Baron, R. M., \& Kenny, D. A. (1986). The moderator-mediator variable distinction in social psychological research: Conceptual, strategic, and statistical considerations. Journal of Personality and Social Psychology, 51(6), 1173-1182. doi: 10.1037/0022-3514.51.6.1173.

Battilana, J., \& Casciaro, T. (2013). Overcoming Resistance to Organizational Change: Strong Ties and Affective Cooptation. Management Science, 59(4), 819-836.
Baumeister, R. F., Gailliot, M. T., DeWall, C. N., \& Oaten, M. (2006). Self-regulation and personality: Strength-boosting interventions and trait moderators of ego depletion. Journal of Personality and Social Psychology, 74, 1773-1802.

Bentler, P. M. (1989). EQS, Structural Equations, Program Manual, Program Version 3.0, Los Angeles: BMDP Statistical Software, Inc.

Bordia, P. Restubog, S. L. D., Jimmieson, N., \& Irmer, B. (2011). Haunted by the past: Effects of poor change management history on employee attitudes and turnover. Group \& Organization Management, 36(2) 191-222.

Bouckenooghe, D. (2010). Positioning Change Recipients'Attitudes Toward Change in the Organizational Change Literature. The Journal of Applied Behavioral Science, 46(4), 500-531.

Bortolotti, L. V., Souza Junior, A. F. d., \& Andrade, D. F. D. (2009, outubro). Uma proposta de modelo para avaliar a resistência à mudança organizacional. Paper presented at the Anais do VI Simpósio de Excelência em Gestão e Tecnologia (SEGET), Resende, RJ.

Bovey, W. H., \& Hede A. (2001). Resistance to organizational change: the role of cognitive and affective processes. Leadership \& Organization Development Journal, 22(8), 372-382.

Coyle-Shapiro, J. A.-M. (1999). Employee participation and assessment of an organizational change intervention: a three wave study of Total Quality Management. Journal of Applied Behavioral Science, 35(4), 439-456.

Choi, M. (2011). Employees' attitudes toward organizational change: A literature review. Human Resource Management, 50(4), 479-500. doi: 10.1002/hrm.20434

Cunningham, G. B. (2006). The relationships among commitment to change, coping with change, and turnover intentions. European Journal of Work and Organizational Psychology, 15(1), 29-45.

Devos, G., Buelens, M., \& Bouckenooghe, D. (2007). The contribution of content, context, and process in understanding openness to organizational change: Two experimental simulation studies. Journal of Social Psychology, 147(6), 607-629.

Eilam, G., \& Shamir, B. (2005). Organizational Change and SelfConcept Threats. The Journal of Applied Behavioral Science, 41(4), 399-421. doi: 10.1177/0021886305280865

Fischer, H. C. R., \& Lima, S. M. V. (2005). Validação de instrumento para diagnóstico de condições facilitadoras de mudança organizacional. Revista Psicologia, 5, 13-44.

Ford, J. D., Ford, L. W., \& D'Amelio, A. (2008). Resistance to change: The rest of the story. Academy of Management Review, $33(2), 362-377$.

George, J. M., \& Jones, G.R. (2001). Towards a process model of individual change in organization. Human Relations, 54(4), 419-444.

Hatch, M. J., \& Ehrlich, S. B. (1993). Spontaneous humor as an indicator of paradox and ambiguity in organizations. Organization Studies, 14, 505-26.

Herscovitch, L., \& Meyer, J. P. (2002). Commitment to organizational change: Extension of a Three-component Model. Journal of Applied Psychology, 87(3), 474-487.

Hodgkinson, G. P., \& Healey, M. P. (2008). Cognition in organizations. Annual Review of Psychology, 59, 387-417. 
Huy, Q. N. (2002). Emotional Balancing of Organizational Continuity and Radical Change: The Contribution of Middle Managers. Administrative Science Quarterly, 47, 31-69.

Isabella, L. A. (1990). Evolving interpretations as a change unfolds: how managers construe key organizational events. Academy of Management Journal, 33(1), 7-41.

Kalimo, R., Taris, T. W., \& Schaufeli, W. B (2003). The effects of past and anticipated future downsizing on survivor wellbeing: an equity perspective. Journal of Occupational Health Psychology, 8(2), 91-109.

Kim, T. G., Hornung, S., \& Rousseau, D. M (2011). Changesupportive employee behavior: antecedents and the moderating role of time. Journal of Management, 37, 1664-1693.

Kruglanski, A. P., A., Higgins, E., \& Capozza, D. (2007). "On the move" or "staying put": Locomotion, need for closure, and reactions to organizational change. Journal of Applied Social Psychology, 37(6), 1305-1340.

Lau, C. M., \& Woodman, R. (1995). Understanding organizational change: a schematic perspective. Academy of Management Journal, 38(2), 537-554.

Lima, S. M. V., \& Bressan, C. L. (2003). Mudança Organizacional: uma introdução. In S. M. V. Lima (Ed.), Mudança Organizacional: teoria e gestão (pp. 17-63). Rio de Janeiro FGV.

Lima Filho, D. O., Sproesser, R. L., \& Hoffmann, G. H. (2005). Reforma administrativa e resistências externas: um estudo de caso. Revista de Administração Pública, 39(1), 123-13.

Lines, R. (2005). The Structure and Function of Attitudes Toward Organizational Change. Human Resource Development Review, 4, 8-32. doi: 10.1177/1534484304273818

Meyer, J. P., Srinivas, E. S., Lal, J B., \& Topolnytsky, L. (2007). Employee commitment and support for an organizational change: Test of the three-component model in two cultures. Journal of Occupational and Organizational Psychology, 80(2), 185-211.

Neiva, E. R., Ros, M., \& Paz, M. G. T. (2005). Attitudes towards organizational change: validation of a scale. Psychology in Spain, 9(1), 81-90.

Neiva, E. R., \& Brito, M. J. P. (2008). Redes sociais e mudança em uma associação de produtores rurais. Psicologia (Florianópolis), 8, 5-24.

Neiva, E. R., \& Paz, M. G. T. d. (2012). Percepção de mudança individual e organizacional: o papel das atitudes, dos valores, do poder e da capacidade organizacional. Revista de Administração (São Paulo), 47, 22-37.

Neiva, E. R., \& Nery, V. F (2012, july). Change reactions: The role of change context and attitudes toward change. Paper presented at the XXX International Congress of Psychology. In Abstracts of the XXX International Congress of Psychology, International Journal of Psychology, Volume 47, Cape Town, South Africa.

Neiva, E. R., Goulart, P. A. R., Fussi, C. C., \& Nery, V. F. (2012, setembro). Contexto e atitudes na mudança organizacional: um estudo realizado em quatro organizações brasileiras. In: XXXVI Encontro da ANPAD, 2012, Rio de Janeiro. Anais do XXXVI Encontro da ANPAD, 2012. v. 1. p. 1-17.
Nery, V. F., \& Neiva, E. R. (2012, maio). Construção e validação do inventário de reações comportamentais à mudança organizacional. V Congresso Brasileiro de Psicologia Organizacional e do Trabalho. In: Anais do V Congresso Brasileiro de Psicologia Organizacional e do Trabalho, Rio de Janeiro/ RJ, Universidade do Estado do Rio de Janeiro UERJ, Brasil.

Oreg, S. (2003). Resistance to Change: Developing an Individual Differences Measure. Journal of Applied Psychology, 88(4), 680-693.

Oreg, S., Vakola, M., \& Armenakis, A. (2011). Change Recipients' Reactions to Organizational Change: A 60-Year Review of Quantitative Studies. The Journal of Applied Behavioral Science. doi: 10.1177/0021886310396550

Pettigrew, A. M., Woodman, R. W., \& Cameron, K. S. (2001). Studying organizational change and development: challenges for future research. Academy of Management Journal, 44(4), 697-713.

Piderit, S. K. (2000). Rethinking resistance and recognizing ambivalence: A multidimensional view of attitudes toward an organizational change. Academy of Management Review, 25(4), 783-794.

Rafferty, A. E., \& Griffin, M. A. (2006). Perceptions of Organizational Change: A Stress and Coping Perspective. Journal of Applied Psychology, 91(5), 1154-1162.

Santos, J. N., Neiva, E. R., \& Andrade-Melo, E. A. (2013). Relação entre clima organizacional, percepção de mudança organizacional e satisfação do cliente. Psicologia: Teoria e Pesquisa, 29, 31-39.

Self, D., Armenakis, A., \& Schraeder, M. (2007). Organizational change content, process and context: A simultaneous analysis of employee reactions. Journal of Change Management, 7(2), 211-229.

Trader-Leigh, K. E. (2002). Case study: Identifying resistance in managing change. Journal of Organizational Change Management, 15, 138-155.

Vakola, M., Tsaousis, L., \& Nikolaou, L. (2004). The role of emotional intelligence and personality variables on attitudes toward organizational change. Journal of Managerial Psychology, 19(2), 88-110.

Valley, K. L., \& Thompson, T. A. (1998). Sticky ties and bad attitudes: relational and individual bases of resistance to change in organizational structure. In R. M. Kramer \& M. A. Neale (Eds.), Power and influence in organizations (pp. 39-66). London: Sage publications, Inc.

Zamuto, R., \& O'Connor E. (1992). Gaining advanced manufacturing technologies benefits: the role of organizational design and culture. Academy of Management Review, 17, 701-728.

Recebido em 29.06.2013

Primeira decisão editorial em 28.02.2014

Versão final em 24.04.2014

Aceito em 23.10.2014 